\title{
HOMOGAMIA EDUCACIONAL EN \\ MÉXICO Y BRASIL, 1970-2000: \\ PAUTAS Y TENDENCIAS*
}

\author{
Albert Esteve \\ Universitat Autònoma de Barcelona
}

\author{
Robert McCaa \\ University of Minnesota \\ Received 12-01-2005; Revise and Resubmit 1-19-2006; \\ Revised Received 4-11-2006; Final Acceptance 4-26-2006
}

\begin{abstract}
Resumen: En un contexto histórico de expansión educativa, mejora de los rendimientos de la educación y aumento de la participación de la mujer en la actividad económica, este artículo examina y compara las pautas y tendencias en homogamia educativa en México y Brasil entre 1970 y 2000. Concretamente, tratamos en perspectiva temporal y comparada las siguientes cuestiones: grado y alcance de la homogamia educativa y simetría en las relaciones de género. Para ello utilizamos las muestras armonizadas de microdatos de los censos de México 1970, 1990 y 2000, y de Brasil 1970, 1980, 1991 y 2000, puestas a disposición por el proyecto IPUMS-International. Los resultados muestran un aumento de la homogamia entre las capas más instruidas y una disminución de la hipergamia femenina en ambos países. Comparativamente, la homogamia educativa es mayor en Brasil que en México, reflejo de una mayor desigualdad social, mientras que las diferencias de género son mayores en México.
\end{abstract}

\section{INTRODUCCIÓN}

La composición de las parejas está sujeta a los principios básicos que articulan el conjunto de las relaciones sociales, entre ellos el principio de homofilia, aquel por el cual personas con similares características se unen entre ellas en una proporción mayor que entre personas disimilares (McPherson et al. 2001). La plasmación de la homofilia en el proceso

*Una versión de este trabajo fue presentada en la conferencia de la International Union for the Scientific Study of the Population, celebrada en Tours, Francia, en 2005. Esta investigación fue financiada, en parte, por fondos de National Institutes of Health Grant No. R01 HD044154-01 y National Science Foundation Grant No. SBR-9908380. 
de selección conyugal da lugar a las uniones homógamas. Para mayor precisión y dado que en este trabajo utilizaremos recurrentemente los conceptos de homogamia, hipergamia e hipogamia, creemos oportuno detenernos en su definición. El vocablo 'homogamia' está compuesto por el sufijo de origen griego 'gamos', que significa 'unión' o 'matrimonio', y por el prefijo del mismo origen 'homo', que significa 'igual' o 'mismo'. La combinación de 'homo' y 'gamos' da como resultado 'unión entre iguales'. En ciencias sociales, concretamente en sociología, demografía y antropología, 'homogamia' se utiliza para designar aquellas uniones o matrimonios entre personas con características en común, básicamente de tipo social o adquirido. Lo contrario de la homogamia es la heterogamia. Entre las parejas heterógamas distinguimos entre 'hipergamia' e 'hipogamia' cuando la variable a partir de la cual examinamos la similitud o diferencia entre los cónyuges es una variable jerárquica, como es el caso de la educación. Esta distinción está basada en el hecho de si es el hombre o la mujer quien ocupa la posición más elevada en dicha jerarquía. En el caso de la educación y tomando la posición de la mujer como referencia, cuando una mujer se casa o se une hacia arriba ('hiper'), con un hombre de mayor nivel de estudios, forma una pareja hipérgama. Cuando una mujer se casa o se une hacia abajo ('hipo'), forma una pareja hipógama.

Acotada la terminología y retomando la cuestión de las uniones, cuando, por ejemplo, dos personas de distinta procedencia étnica se unen, la unión resultante tiende a erosionar entre las generaciones venideras el sentido de pertinencia grupal y a disminuir las actitudes negativas hacia los demás grupos. Con la misma lógica, dada la correlación que existe entre el nivel de educación y el estatus socioeconómico, especialmente en sociedades en desarrollo, la interacción entre grupos educativos es leída en clave de grado y rigidez de estratificación social, aunque, como es lógico, el análisis de la desigualdad social, que es una cuestión de naturaleza caleidoscópica, requiere además identificar sus principales dimensiones, examinar la intensidad y trascendencia de la misma, y explorar su rigidez en base a las oportunidades que genera para la movilidad social. En este contexto, el matrimonio es uno de los ámbitos que mejor refleja el alcance de la desigualdad social, puesto que en él no sólo fraguan las pautas que gobiernan el proceso de selección conyugal, sino las que gobiernan el conjunto de la sociedad. Y el nivel de estudios, ya sea porque manifiesta tanto la desigualdad de oportunidades de formación de los individuos como sus perspectivas de futuro en relación al estatus social o el nivel de ingresos, es una de las variables que mejor traduce el comportamiento diferencial de los individuos en un amplio elenco de dimensiones. Más aun en países como México y Brasil, donde todavía hoy la desigualdad social sigue reproduciéndose a través del acceso desigual a la educación (Mier, Rocha, Rabell 2003; Marteleto 2004). Dos países en los que el estudio de la homogamia educativa adquiere especial 


\section{8}

interés por ser, entre otros aspectos, resultado y fuente de desigualdades sociales y de género. Con todo, sea o no sea la homogamia educativa un buen indicador de estratificación social, las pautas y tendencias en homogamia educativa reflejan los cambios en la rigidez de las fronteras sociales y son un componente importante de las transformaciones en la composición de las parejas (Schwartz y Mare 2005).

El trabajo que aquí se presenta se enmarca en un periodo histórico, el de las última tres décadas, caracterizado por una fuerte expansión educativa, resultado de la aplicación de programas y medidas gubernamentales para universalizar el acceso a la educación primaria, potenciar la educación secundaria y combatir la desigualdad según condición de residencia urbana o rural, género y pertenencia étnica. Por otro lado, los rendimientos a la educación son cada vez mayores. Invertir en la educación de los hijos resulta cada vez más beneficioso desde el punto de vista de los padres. La mayor escolarización ha contribuido al aumento de las tasas de actividad femenina (García Oliveira 1994), especialmente entre los niveles más instruidos, lo que a su vez, ha mejorado la utilidad de los recursos dedicados a la educación de las hijas, reduciendo así las diferencias de género en materia educativa (Parker y Perdezini 1999; Marteleto 2004).

En este contexto de expansión educativa, mejora de los rendimientos de la educación y aumento de la participación de la mujer en la actividad económica, este artículo examina y compara las pautas y tendencias en homogamia educativa en México y Brasil durante las últimas tres décadas. En concreto, trataremos en perspectiva temporal y comparada las siguientes cuestiones: grado y alcance de la homogamia educativa y simetría en las relaciones de género. Para ello utilizamos las muestras armonizadas de microdatos para los censos de México 1970, 1990 y 2000, y de Brasil 1970, 1980, 1991 y 2000, puestas a disposición por el proyecto Integrated Public Use Microdata Series International (IPUMS-International).

En anteriores trabajos, los autores han examinado las pautas de homogamia educativa para el caso de México (Esteve 2005) a partir de los microdatos censales y una metodología análoga a la que proponemos aquí. En este artículo retomamos el caso de México y lo extendemos al caso de Brasil con el objetivo de explorar las pautas de homogamia educativa en ambos países y proponer argumentos para la comparación y la reflexión. Los datos de ambos países son sometidos a un mismo tratamiento estadístico para extraer resultados comparables.

El artículo se estructura en tres partes más conclusiones. En la primera parte esbozamos los principales argumentos teóricos que nos inclinan a pensar que existe una tendencia hacia una mayor homogamia. En la segunda parte presentamos en detalle los datos utilizados y justificamos la construcción de los grupos educativos, enfatizando la oportunidad que los datos proporcionados por el proyecto IPUMS-International representa para 
llevar a cabo estudios comparativos a escala internacional. En la tercera parte presentamos y analizamos los resultados obtenidos, con especial atención a los resultados de los modelos de homogamia educativa.

\section{HACIA UNA MAYOR HOMOGAMIA EDUCATIVA:} CONSIDERACIONES TEÓRICAS

Existe un amplio consenso entorno al hecho que individuos con similares características tienden a unirse entre ellos, lo que, desde la lógica de las probabilidades, equivale a decir que existen más uniones endógamas y/o homógamas de las que resultarían si los individuos nos emparejáramos al azar. Esta homogeneidad, que valida el principio general de homofilia, ha sido abordada en base a una amplia gama de características, adscritas y adquiridas, entre los que se destacan la religión (Johnson 1980), la etnia (Pagnini y Morgan 1990, McCaa 1993), el estatus socioeconómico (Kalmijn, 1991), la educación (Mare 1991, Blackwell 1998, Smits 2003), y la ocupación (Hout 1982).

La mayor o menor influencia de estas características en la selección de las parejas ha dado lugar a diversas formulaciones teóricas. La pulsión entre lo adscrito y lo adquirido ha sido objeto de debate entre los científicos sociales. La expansión educativa junto al desarrollo económico confiere al nivel de instrucción, característica adquirida por excelencia, un papel preponderante en la configuración de las preferencias conyugales que va asociado a una pérdida de importancia de los aspectos adscritos. A su vez, en las sociedades contemporáneas, dónde la elección del cónyuge es un acto eminentemente voluntario, asistimos al deterioro de la influencia de la familia, la iglesia y el estado en su rol sancionador de las uniones. Pero la posición social y el lugar de residencia siguen ejerciendo su influencia y limitando, por tanto, la oferta de candidatos potenciales con los que formar pareja. En otras palabras, la composición social del vecindario o de los espacios vitales de los individuos-ocio, escuela, trabajo, familia-filtran a los cónyuges potenciales.

Desde la lógica interna del matrimonio, utilizando el lenguaje de los economistas, los científicos han tratado, por un lado, el costo de oportunidad de unirse o no unirse y, por otro, el costo de oportunidad de hacerlo con uno $u$ otro candidato. No en vano, en demografía por ejemplo, nupcialidad y homogamia se tratan por separado. Según la teoría formulada por el economista Gary Becker (1974), que se podría denominar "tradicional", el matrimonio es una institución en la que los cónyuges se complementan en base a una repartición de las tareas domésticas en función del sexo. Los hombres se especializan en las tareas productivas y las mujeres en las reproductivas. Este modelo asume que las preferencias conyugales son asimétricas entre hombres y mujeres. Las mujeres valoran en el hombre su capacidad de éxito en la esfera 
productiva, altamente correlacionada con su nivel de formación. Y los hombres valoran en la mujer su procedencia social, religión, o pertenencia étnica entre otras. La asimetría en las preferencias justificaría el patrón clásico de hipergamia femenina, aquél en el que la mujer tiene un nivel de instrucción menor al de su marido.

Ahora bien, la fórmula tradicional del matrimonio expresada por Becker se enfrenta a serios desafíos ante la incorporación de la mujer al mercado de trabajo, la expansión educativa y la reducción de las diferencias de género en materia educativa. Sin cuestionar explícitamente los beneficios asociados al matrimonio, Oppenheimer (1988) argumenta que el aumento de la independencia económica de las mujeres tendrá un efecto en la selección conyugal, puesto que estas aumentarán su nivel de exigencia, retrasando, si es preciso, la edad al matrimonio hasta encontrar un candidato apropiado. Este cambio erosionará la tradicional asimetría entre hombres y mujeres en relación a sus preferencias en la selección conyugal. Ambos sexos enfrentarán el matrimonio rodeados de parecida incertidumbre, asociada la transición a la etapa adulta y a las perspectivas de una carrera laboral aún incierta. Ante esta incertidumbre, la educación actuará de excelente referente para evaluar el porvenir de los candidatos. En la misma línea, Parrado (2002) muestra para México que el nivel de instrucción es una variable relevante en la selección de la pareja para los hombres y las mujeres. En resumen, si la preferencia por los más instruidos se observa en ambos sexos por igual, la homogamia educativa aumentará: los más educados se unirán entre ellos porque gozan de una mejor posición en el mercado, lo que inducirá al resto de los grupos a un comportamiento similar.

La mayor permanencia en el sistema educativo, asociada a la expansión educacional también resultará en una mayor homogamia educativa. La escuela, en sentido amplio, es un mercado matrimonial excelente, puesto que reúne personas de distinto sexo, mismas edades y similares inquietudes. Al aumentar los años de permanencia en el sistema educativo, la probabilidad de encontrar pareja entre los compañeros de estudio aumenta, especialmente entre los universitarios. Cuanto mayor es la distancia temporal entre la salida del sistema educativo y la entrada en una unión, menor es la probabilidad de formar una unión homógama (Mare 1991). Si el retraso de la edad al matrimonio es el resultado de una mayor permanencia en el sistema educativo, la homogamia educativa aumentará entre aquellos grupos educacionales que permanezcan por un periodo más largo en el sistema, es decir, entre los más instruidos. En conclusión, voluntad y oportunidad se alían con los más instruidos favoreciendo las uniones entre ellos. Varios estudios sostienen la existencia de una relación entre el lapso de tiempo que transcurre entre dejar los estudios y formar matrimonio y la evolución de la homogamia educativa. Es el caso, por ejemplo, del trabajo sobre Irlanda y Gran Bretaña en 
el que Halpin y Chan (2003), inspirados en resultados previos para los Estados Unidos (Mare 1991), llegan a la conclusión de que la diferencia en la edad al matrimonio entre Irlanda y Gran Bretaña es la explicación más probable para justificar las diferencias observadas entre ambos países en los niveles de homogamia educativa. En un estudio reciente sobre la evolución de la homogamia educativa en Estados Unidos entre 1960 y 2000, Schwartz y Mare (2005) muestran que la relación entre homogamia educativa y el lapso de tiempo entre dejar los estudios y el matrimonio no se observa en la dirección esperada en todo el período y que, en cualquier caso, esta relación se observa principalmente en la población con estudios universitarios, mientras que dicha relación no es tan evidente en el resto de las categorías educativas.

Los supuestos teóricos esbozados aquí han sido examinados, y en la mayoría de ocasiones validados, en países desarrollados como los Estados Unidos, España, Reino Unido, y Noruega (Mare 1991; Birkelund y Heldald 2003; Esteve y Cortina 2006; Ni Bhrolchain 2002). Son escasos los estudios que tratan la homogamia educativa en países en vías de desarrollo con índices de escolarización relativamente bajos y acentuadas desigualdades sociales. Son pocos los trabajos sobre el tema en México y Brasil (Solís 2003; Esteve 2005), y se encuentran pocos estudios comparativos a escala internacional. Entre ellos, se encuentra el trabajo de Smits (2003) que argumenta, en base a la experiencia de medio centenar de países con distintos niveles de desarrollo, que existe una relación entre homogamia educativa y nivel de desarrollo. En los países en desarrollo, la homogamia educativa aumentará como resultado de la importancia que la educación adquiere en un contexto de crecimiento económico. Pero cuando los niveles de bienestar se generalicen al conjunto de la población, y la mayoría de la población alcance los niveles superiores del sistema educativo, la correlación entre nivel de instrucción y posición socio-económica se deteriorará, es decir que la educación dejará de ser un referente en el proceso de selección conyugal. Pero en México y Brasil, la generalización de los niveles de bienestar está lejos de ser alcanzada, razón por la cual es importante explorar cómo las transformaciones acontecidas en las últimas décadas han modificado las pautas de homogamia entre distintos grupos.

Hemos dejado para el final de este apartado un aspecto importantísimo que condiciona todo lo dicho hasta el momento entorno a cómo se articulan las preferencias en el proceso de selección conyugal. En efecto, la materialización en la composición de las parejas de las hipótesis esbozadas aquí está sujeta a la demografía de los candidatos y candidatas a formar pareja. Las preferencias individuales deben plasmarse en un mercado finito que condiciona las posibilidades de satisfacerlas. Por ejemplo, si la formación universitaria fuese mayor entre los hombres, éstos tendrían dificultades de emparejarse dentro de su mismo grupo en caso que así 
lo deseara la mayoría, puesto que no habría suficientes candidatas del mismo perfil. A su vez, si la estructura educativa de la población estuviese excesivamente concentrada, los niveles de homogamia serían imperativamente mayores que si esta estructura estuviese mucho más diversificada. La interpretación de los niveles de homogamia no puede hacerse al margen de los determinantes estructurales que impone la oferta y la demanda de candidatos y candidatas en el mercado matrimonial. Con el objetivo de aislar los efectos específicos de homogamia de los efectos específicos de la oportunidad, recurrimos a los modelos loglineales. Ahora bien, aislados los efectos específicos de homogamia, éstos no pueden atribuirse en exclusiva a una cuestión de preferencias, puesto que pueden ser también el resultado de un proceso de competencia en el mercado. Por ejemplo, si la educación universitaria es especialmente preciada entre hombres y mujeres indistintamente de su nivel de instrucción, serán los hombres y mujeres con estudios universitarios los que se unan preferentemente entre sí, induciendo al resto de los grupos a un comportamiento similar. En esta situación, los niveles específicos de homogamia serán altísimos para todos los grupos, pero estos no podrán atribuirse exclusivamente a una cuestión de preferencias.

\section{DATOS Y MÉTODOS}

\section{Adecuación de las muestras}

Los datos utilizados en esta investigación proceden de las muestras de microdatos censales armonizadas puestas a disposición por el proyecto IPUMS-International, con sede en el Minnesota Population Center. En concreto, se trata de las muestras de microdatos de los censos brasileños de 1970, 1980, 1991 y 2000, y de los censos mexicanos de 1970, 1990 y 2000 , de densidad variable entre el $1 \%$ y el $10 \%$.

Los microdatos censales constituyen una fuente de valor incomparable para el estudio en ciencias sociales. Aunque otras fuentes sociodemográficas ofrecen mayor nivel de detalle conceptual, ninguna ofrece tal grado de cobertura territorial y temporal con mayor densidad muestral. Los censos ofrecen información sociodemográfica básica de los individuos (edad, sexo, estado civil, lugar de nacimiento, etc.) junto con información de los hogares y las parejas que, gracias a que las muestras de microdatos están organizadas por hogares, pueden trabajarse simultáneamente.

La comparación internacional en base a microdatos censales es poco frecuente, en parte, porque el acceso a los microdatos está restringido o 
es difícil de obtener. En este sentido, el proyecto IPUMS-International facilita el acceso a los datos armonizados de forma gratuita y a través de Internet (https:/ / www.ipums.org/international).

Los microdatos contienen registros individuales organizados por hogares. La estructura del hogar es fundamental para poder recomponer el archivo de individuos original en un archivo de parejas. Para establecer el vínculo entre los cónyuges, IPUMS-International ofrece la variable SPLOC (spouse location), que identifica el cónyuge para todas aquellas personas de un hogar, sean o no jefe del hogar, cuyo cónyuge se encuentre entre las personas que lo integran. SPLOC utiliza la relación de parentesco y, cuando está disponible, información adicional sobre los distintos núcleos familiares. Sin embargo, en hogares con múltiples núcleos familiares, la asignación del cónyuge es compleja y, a menudo, con la información utilizable, no es posible identificar con la misma precisión quién es cónyuge de quién en aquellas uniones en las que no interviene directamente la persona principal, hecho que podría acarrear algún tipo de sesgo, que no estimamos muy significativo dado el reducido número de casos en los que esto ocurre.

A partir de la variable estado civil, clasificamos las parejas según estén unidas en matrimonio o formen una unión consensual. Del total de parejas, en nuestro análisis trabajamos sólo con aquéllas en las que como mínimo uno de los cónyuges tiene entre 30 y 39 años de edad. Con la selección de un grupo decenal de edades evitamos el solapamiento de las cohortes de un censo a otro. Trabajar con el grupo 30-39 minimiza la selección de calendario porqué, a los 30 años, la proporción de alguna vez casados/as o unidos / as es muy cercana a la proporción final de las generaciones implicadas. Seleccionando edades más tempranas existe el riesgo de no incluir a los individuos que están retrasando la edad al matrimonio o unión, muy probablemente por causas relacionadas con su formación académica. Por otra parte, a estas edades el efecto de la disolución de las uniones por divorcio o mortalidad también se minimiza.

La estructura final de los datos sometida al análisis es relativamente simple (ver Apéndices 1 y 2). Se trata de la clasificación de las parejas seleccionadas en función del tipo de unión (casados y unidos), año (Brasil: 1970, 1980, 1991, 2000, y México: 1970, 1990, 2000), educación del hombre (5 grupos) y educación de la mujer (5 grupos). En esta clasificación aparece el total de parejas de la muestra ponderada pero no expandida al total de la población. En aras a la ejecución de los modelos, la muestra expandida aumenta innecesariamente el número de casos en ya de por sí muestras con un elevado número de casos, al tratarse de muestras censales de altas densidades ${ }^{2}$.

2. Conforme aumenta el número de casos, los estadísticos que miden la bondad de ajuste de los modelos tienden a priorizar los modelos más complejos, sin aportar información 


\section{Latin American Research Review}

La inclusión en el análisis de otras variables, tales como raza, urbanorural o religión, por citar sólo una selección de las más representativas, enriquecería, sin duda alguna, los resultados, pero también añadiría mayor dificultad a la especificación de los modelos de homogamia y complicaría la comparación entre países, alejándonos de los objetivos planteados en este trabajo. Respecto a la edad al matrimonio, las muestras de microdatos utilizadas no contienen esta información, razón por la cual no podemos usar esta variable para examinar la validez de la hipótesis que relaciona edad al matrimonio con el nivel de homogamia educativa.

\section{Nivel de instrucción}

El nivel de instrucción tomado como referencia es el declarado en la fecha censal y, por tanto, no se corresponde con el que tenían los cónyuges en el momento de casarse o unirse. Sin embargo, no consideramos que esta decisión, obligada por la disponibilidad de los datos, afecte en demasía a los resultados finales, puesto que el nivel de instrucción adquirido apenas varía después de la celebración del matrimonio, produciéndose éste, de manera mayoritaria, al concluir la etapa formativa.

Asumiendo, por tanto, que la captación del nivel educativo en el momento censal no supone un sesgo significativo, lo que resulta verdaderamente importante es obtener una clasificación del nivel educativo apropiada para el análisis y, sobretodo, apta para la comparación entre países. En efecto, la creación de una clasificación por nivel educativo comparable entre México y Brasil es uno de los retos de esta investigación. De entrada, los países estudiados no comparten los mismos sistemas educativos; en base a los años de escolarización, el sistema mexicano presenta sus principales divisiones a los 6 años (Primaria), a los 9 (Secundaria Elemental) a los 12 (Secundaria) y a los 16 (Estudios Superiores); en Brasil las divisiones se observan a los 4 años de escolarización (Primaria), a los 8 (Secundaria básica), a los 11 (Secundaria superior) y a los 15 (Estudios Superiores). Una dificultad añadida es que algunos censos no recogen con precisión todos los niveles del sistema educativo. Es el caso, por ejemplo, del censo de Colombia de 1993, que no informa de los que han finalizado sus estudios universitarios, razón por la cual Colombia ha quedado fuera de este análisis pese a formar parte de la base de IPUMS-International.

La base de datos IPUMS-International ofrece dos variables sobre el nivel educativo: el nivel educativo alcanzado (EDATTAIN) y los años de escolarización (YRSCHOOL). En el primer caso, EDATTAIN registra

adicional que en lo sustancial modifique las conclusiones que se extraen de modelos más sencillos pero que, debido al elevado número de casos, no presentan un buen ajuste según estos mismos estadísticos. También, al aumentar el número de casos, condicionamos el calculo de la significación de los parámetros, aunque, tratándose de muestras censales, la preocupación por la significación estadística queda relegada a un segundo plano. 
el nivel educativo completado o por el que se ha obtenido un diploma, es decir, si alguien ha cursado tres de los cuatro cursos para obtener el diploma universitario, la variable EDATTAIN considera que el máximo nivel completado para esta persona es secundaria. EDATTAIN pretende ser comparable entre países y para ello adopta la clasificación estándar de Naciones Unidas que establece la primaria en 6 años, la secundaria elemental en 3 y la secundaria superior en 3. EDATTAIN aplica este esquema a aquellas muestras que sólo proporcionan el número de años de escolarización ${ }^{3}$.

La segunda variable educativa disponible en la base de datos IPUMS es YRSCHOOL. Esta variable indica en años el total de cursos superados en el sistema educativo. La principal ventaja de YRSCHOOL en relación a EDATTAIN es que permite reconstruir las distintas categorías educativas libremente, por ejemplo, de forma independiente para cada país, de acuerdo con su propio sistema educativo. Por esta razón hemos tomado YRSCHOOL como variable de referencia. En base a esta variable, en México se han creado 5 categorías: menos de 6 años de escolarización, 6-8, 9-11, 12-15, y 16 o más. Para Brasil se crean también 5 grupos aunque con distinta periodización: menos de 4, 4-7, 8-10, 11-14, y 15 o más. Estas categorías son, así, distintas para cada país pero, sin embargo, reflejan esencialmente las mismas divisiones educativas en ambos casos: menos de primaria, primaria, secundaria elemental, secundaria superior y estudios superiores.

La clasificación finalmente adoptada condiciona, como lo haría cualquier otra, el resultado de los indicadores y los modelos que se calculan sobre su base. Antes de decidirnos por esta clasificación, tanteamos distintas alternativas. Por ejemplo, para poder incluir Colombia en el trabajo, decidimos combinar los grupos 'secundaria completa' y 'estudios universitarios' en una sola categoría para todos los países y años, puesto que el censo de 1993 de Colombia no ofrece esta distinción. Los niveles resultantes de homogamia fueron altamente sensibles a esta modificación, hecho que demuestra que poseer estudios universitarios es una distinción relevante y significativa que obliga a considerar independientemente a los universitarios como grupo. Lo mismo ocurre por la parte baja de la jerarquía educativa. Imponer como mínimo seis años de escolarización para considerar finalizados los estudios primarios significa, en el caso de Brasil, aumentar sustancialmente la población por debajo de este umbral, puesto que en este país existe una divisoria educativa muy marcada a los cuatro años de escolarización. En conclusión, sin obviar la influencia que cualquier clasificación ejerce sobre los resultados, hemos optado por aplicar aquella clasificación que representara mejor la lógica del sistema

3. Para obtener mayores precisiones sobre la construcción de ésta variable consultar: http:// www.ipums.org/international/ \{Variables\} \{EDATTAIN\} 
educativo de cada país exigiendo, no obstante, el mismo número de categorías en cada uno de ellos.

\section{MODELOS LOGLINEALES}

Para aislar los efectos estructurales de los efectos específicos de homogamia educativa, recurrimos a los modelos loglineales. Estos modelos son comúnmente utilizados para analizar las pautas de interacción entre dos o más variables (Knoke y Burke, 1980). A diferencia de los modelos de regresión logística u otras técnicas afines, como el event history analysis, los modelos loglineales poseen algunas características especialmente apropiadas para el análisis de la homogamia. En primer lugar, no asumen una relación de causalidad entre variable dependiente e independiente, sino que miden la asociación entre dos o más variables más allá de lo que se relacionarían por la simple intervención del azar, permitiendo así formulaciones teóricas más flexibles. En segundo lugar, los modelos loglineales tratan el mercado matrimonial en forma holística, es decir, considerando todas las interacciones posibles sin necesidad de fragmentarlas para ser adaptadas a otro tipo de técnicas. Se trata pues de una visión más cercana a los mercados matrimoniales, que no obliga a fracturar el análisis en múltiples combinaciones o transiciones, que, en la mayoría de casos, son interdependientes. En tercer lugar, y no menos importante, los modelos loglineales descomponen jerárquicamente cada uno de los efectos. Por ejemplo, ofrecen parámetros específicos del efecto de pertenecer a un grupo A, el efecto de pertenecer a un grupo $\mathrm{B}$ y el efecto de pertenecer a $\mathrm{A}$ y B simultáneamente. Es precisamente este último efecto el que se utiliza como indicador específico de asociación entre dos grupos, libre del efecto de la estructura o de la distribución de los marginales.

\section{RESULTADOS}

\section{Expansión educativa y disminución de las uniones homógamas}

La figura 1 muestra la distribución de los cónyuges seleccionados para este estudio (parejas en las que al menos uno de sus miembros tiene entre 30 y 39 años de edad en el momento del censo) por nivel de instrucción y sexo para México y Brasil respectivamente. No es necesario ahondar en demasía en la exploración de los datos para percatarse de las sobresalientes transformaciones que acontecen en ambos países. En México, la proporción de individuos con estudios primarios crece significativamente entre 1970 y el año 2000, del 40\% al 80\% para ambos sexos aproximadamente, mientras aumenta la proporción para el resto de grupos educacionales. Los principales aumentos se observan, para ambos sexos, entre las categorías más elevadas (12-15 y 16 o más años de edad). La proporción de 


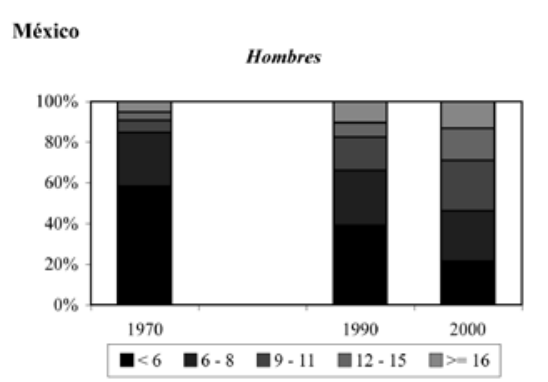

Brasil

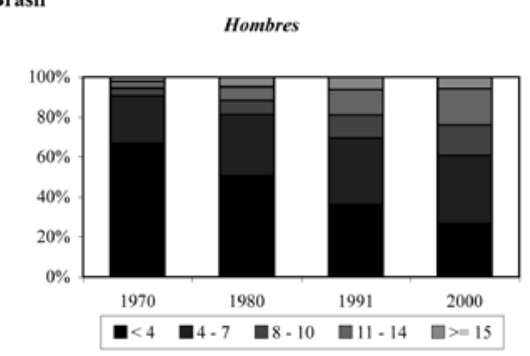

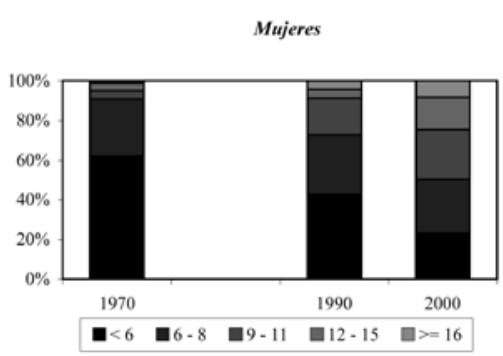

Mujeres

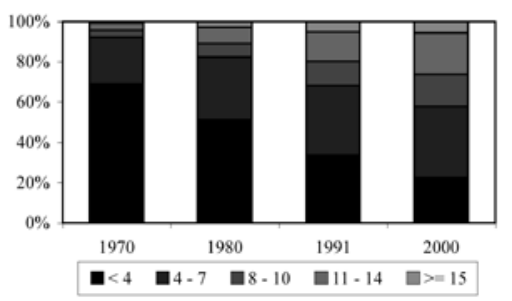

Figura 1 Distribución de los cónyuges por nivel de instrucción, sexo y país, 1970-2000 Fuente: Minnesota Population Center. Integrated Public Use Microdata Series-International: Version 2.0. Minneapolis: University of Minnesota, 2006.

hombres con estudios universitarios aumenta del 5,1\% al 13,2\% entre 1970 y 2000, y en el caso de las mujeres, aumenta del 1,1\% al 8,3\%; un aumento relativamente mayor al de los hombres, lo que contribuye a disminuir las diferencias de género, pero no a eliminarlas.

En Brasil, la proporción de hombres y mujeres con cuatro o más años de educación también aumenta en forma importante, del 33,4\% al 73,5\% para los hombres, y del $31 \%$ al $77,5 \%$ para las mujeres. Consecuentemente, esta reducción conlleva un aumento en el resto de las categorías, especialmente de las más elevadas. Entre 1970 y 2000, la proporción de universitarios aumenta del 2,2\% al 5,8\% para los hombres, y del 0,6\% al $5,7 \%$ para las mujeres; un aumento superior al de los hombres en términos absolutos y relativos, lo que no sólo contribuye a reducir las diferencias de género, sino a hacerlas desaparecer.

Al examinar comparativamente los casos de México y Brasil, salvando las diferencias que la distinta clasificación de los grupos educativos pueda crear, se destacan dos similitudes y dos diferencias importantes. Por un lado, en ambos países la estructura educativa se expande y se desconcentra: el peso de la categoría educacional más baja se reduce a una tercera parte del nivel de 1970. Asimismo, las diferencias de género en materia educativa tienden a desaparecer. Por otro lado, la permanencia en las categorías más elevadas destaca que México tiene una clara desigualdad 


\section{México}

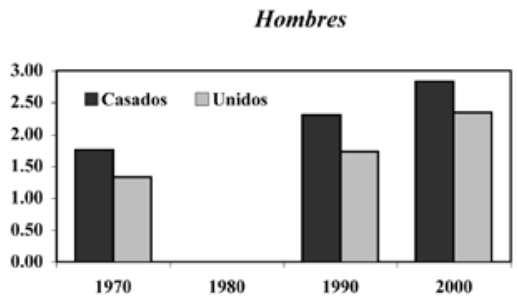

Brasil

Hombres

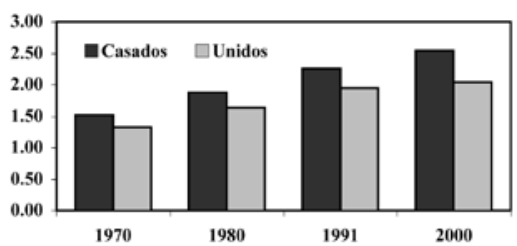

Mujeres

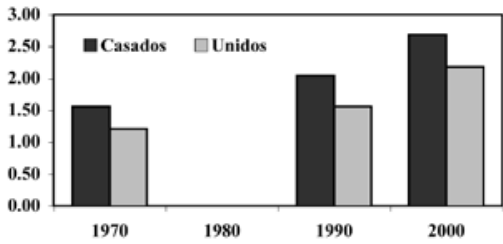

Mujeres

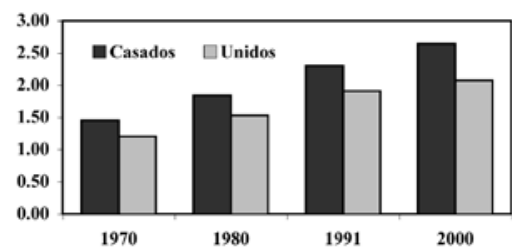

$(0$ = menos de primaria, 1 = primaria; 2 = secundaria básica; 3 = secundaria superior; 4 = universitaria incompleta; $5=$ universitaria)

Fuente: Minnesota Population Center. Integrated Public Use Microdata SeriesInternational: Version 2.0. Minneapolis: University of Minnesota, 2006.

Figura 2 Nivel de instrucción medio por tipo de unión, sexo y país, 1970-2000. (0= menos de primaria, 1 = primaria; $2=$ secundaria básica; $3=$ secundaria superior; $4=$ universitaria incompleta; 5 = universitaria)

Fuente: Minnesota Population Center. Integrated Public Use Microdata Series-International: Version 2.0. Minneapolis: University of Minnesota, 2006.

de género, ausente en Brasil, y una mayor desigualdad en la proporción de población con estudios universitarios a comparación de Brasil. Tal y como señalaremos más adelante, las diferencias observadas entre estos países en la Figura 1 son indicativas de diferencias de mayor alcance.

Los datos de la Figura 1 aparecen resumidos mediante el índice medio de instrucción en la Figura 2, que además distingue por tipo de unión. El cálculo del índice es extremadamente simple: se asigna un valor de 1 a 5 a cada nivel educativo de menor a mayor y se calcula el promedio. Los resultados obtenidos corroboran las pautas comentadas arriba pero añaden que la población unida en matrimonio tiene un nivel de instrucción medio superior a la población en unión consensual. Este patrón se mantiene en el tiempo y no tiende a desaparecer, razón por la cual hemos decidido, a partir de ahora, introducir la distinción por tipo de unión en el análisis.

La figura 3 ilustra la distribución de las parejas según sean homógamas e hipérgamas, o hipógamas desde el punto de vista femenino, por tipo de unión para ambos países. Una pareja hipérgama es aquella en la que la mujer tiene un nivel de instrucción inferior al del hombre; una pareja 


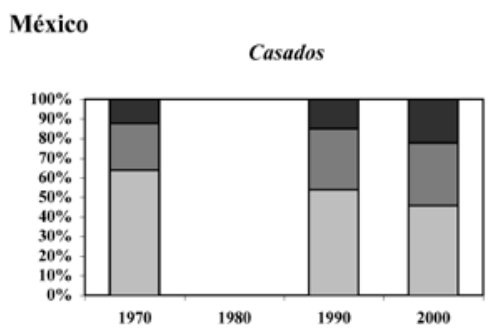

$\square$ Homogamia ${ }^{-H i p e r g a m i a ~ f e m e n i n a ~} \mathbf{E}$ Hipogamia femenina

Brasil

Casados

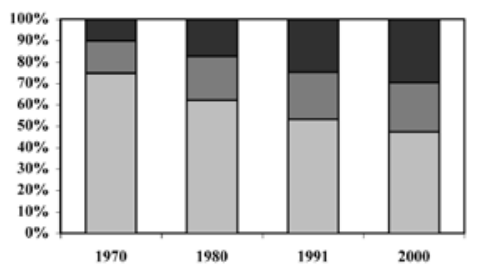

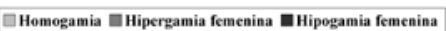

Unidos

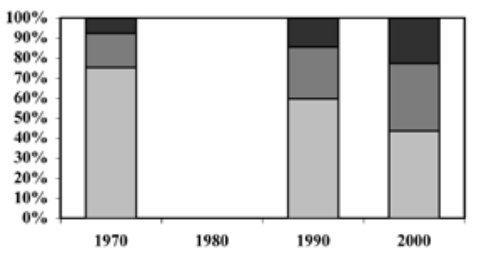

Homogamia $\square$ Hipergamia femenina $\mathbf{H i p o g a m i a ~ f e m e n i n a ~}$

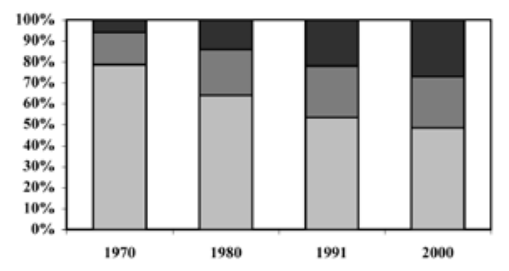

- Homogamia E Hipergamia femenina EHipogamia femenina

Fuente: Minnesota Population Center. Integrated Public Use Microdata Series-International: Version 2.0. Minneapolis: University of Minnesota, 2006.

Figura 3 Distribución de las parejas según condición de homogamia, tipo de unión y país, 1970-2000

Fuente: Minnesota Population Center. Integrated Public Use Microdata Series-International: Version 2.0. Minneapolis: University of Minnesota, 2006.

hipógama es aquella con la situación inversa. Tanto en México como en Brasil, indistintamente del tipo de unión, la proporción de parejas homógamas disminuye en el tiempo, hasta situarse por debajo del $50 \%$. En el año 2000, la proporción combinada de hipergamia e hipogamia supera la proporción de homogamia. La homogamia sigue siendo ligeramente superior en Brasil que en México, fruto probablemente de una mayor concentración de su estructura educativa en los grupos más bajos.

En México, las diferencias de homogamia entre matrimonios y uniones consensuales tienden a desaparecer. En 1970 la proporción de homogamia entre las uniones consensuales es mayor a la misma proporción entre los matrimonios (75,3\% por $63,9 \%$ respectivamente). Sin embargo, en 2000, la proporción de homogamia entre los matrimonios es ligeramente superior a la misma proporción entre las uniones consensuales $(45,7 \%$ por $43,7 \%$ respectivamente). El descenso de la homogamia conlleva un aumento de las parejas hipérgamas e hipógamas. Para todos los años y tipos de unión, la proporción de parejas hipérgamas es superior a la proporción de parejas hipógamas, mostrando que existe una marcada pauta de hipergamia femenina. 
En Brasil, no se observan excesivas diferencias entre matrimonios y uniones en cuanto a sus niveles de homogamia educativa. La proporción de parejas homógamas decrece mientras que, consecuentemente, aumenta la heterogamia. La proporción de parejas hipérgamas es mayor a la proporción de hipógamas en 1970 y en 1980, pero esta situación se invierte en 1991 y 2000. En los últimos años, la proporción de parejas hipógamas es superior a la proporción de hipérgamas. Esta inversión de la pauta en heterogamia no se observa en el caso de México y es, por tanto, una diferencia importante entre los dos países. Si bien la mayor hipergamia femenina observada en México puede atribuirse a una desigual distribución por nivel educativo entre hombres y mujeres, en el caso de Brasil, no existe tal desigualdad.

Ante estos resultados, surgen diversas preguntas. Por un lado, ¿en qué medida la diversificación de la estructura educativa observada en México y Brasil ha ampliado las oportunidades de formar pareja con otros grupos $\mathrm{y}$, por consiguiente, hasta qué punto dicha diversificación es responsable de la caída del nivel global de homogamia? Por otro lado, ¿en qué medida la pauta de hipergamia femenina observada en México para todos los años y en Brasil en 1970 y 1980 es el producto de la distribución desigual por nivel educativo entre hombres y mujeres? Para responder estas preguntas, es preciso aislar el efecto que la mayor o menor concentración de la estructura educativa y las desigualdades de género en esta materia ejercen sobre los niveles de homogamia y heterogamia observados a partir de las proporciones, ya que las mismas son medidas útiles e informativas, pero altamente condicionadas por la estructura.

\section{Especificación de los modelos de homogamia}

Como hemos avanzado en el apartado metodológico, utilizamos los modelos loglineales para examinar las pautas de asociación entre grupos educativos y así controlar el efecto de la estructura educativa de los esposos. Con el objetivo de responder a las preguntas planteadas, examinamos distintas hipótesis, partiendo de la hipótesis de independencia. La independencia asume que no existe relación entre la educación del hombre y la educación de la mujer y que, por tanto, la educación no es una variable a tener en cuenta en la selección de la pareja. Este supuesto equivale a decir que la distribución de las parejas por nivel educativo de los cónyuges es el resultado del azar y sólo estaría condicionado por el número de hombres y mujeres por nivel educativo. Las frecuencias esperadas derivadas de este modelo, en base a una tabla de doble entrada, se estiman según esta expresión:

$$
\log f_{j}=\mu_{0}+\mu_{i}+\mu_{j}
$$




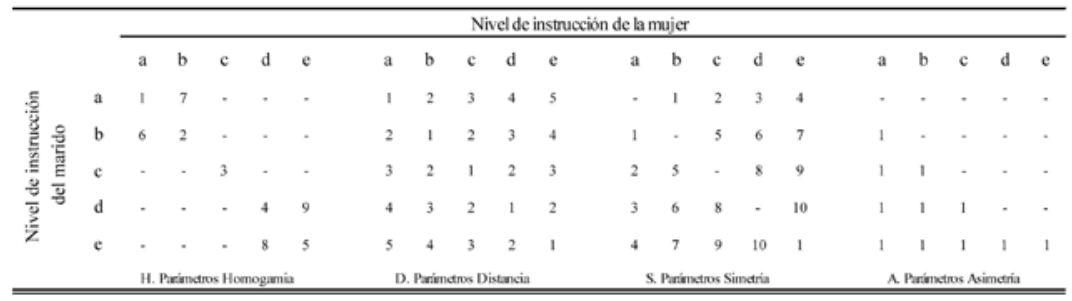

a, menos de primaria; $b$, primaria; $c$, secundaria elemental; $d$, secundaria; $e$, estudios superiores

Cuadro 1 Estructura topológica de las hipótesis de homogamia educativa comprobadas en los modelos

Donde $\log f i_{j}$ es el logaritmo natural de la frecuencia esperada de la fila $i$ columna $j ; \mu_{0}$ la constante; $\mu_{i}$ el parámetro para la fila $i ; \mu_{j}$ el parámetro par la columna $j$.

Si asumimos, en cambio, una asociación específica para cada una de las combinaciones de grupos educativos, año y tipo de unión, obtenemos lo que se conoce como modelo saturado, expresado:

$$
\log f_{j}=\mu_{0}+\mu_{i}+\mu_{j}+\mu_{i j}
$$

Donde $\mu_{i j}$ es el parámetro de la interacción entre la fila $i$ la columna $j$.

El modelo saturado, aunque tiene la particularidad de reproducir exactamente los datos, no tiene interés analítico, puesto que consume tantos parámetros como interacciones quiere explicar. Se trata, por tanto, de un modelo con nula parsimonia. Sin embargo, entre el modelo de independencia y el modelo saturado, existen numerosas especificaciones que, con más o menos parsimonia, tienen interés explicativo en términos sustantivos.

El cuadro 1 muestra gráficamente la estructura topológica de los parámetros básicos para cada una de las principales hipótesis examinadas: Homogamia (H), Distancia (D), Simetría (S) y Asimetría (A). En una tabla de contingencia de doble entrada en la que ambas variables comparten exactamente las mismas categorías, es habitual encontrar que las celdas de la diagonal concentren más casos de los que se concentrarían por azar. Cuando esto ocurre, es apropiado explorar la condición de quasiindependencia, que asume independencia en todas las celdas excepto en las de la diagonal, que recogen las parejas homógamas. La quasiindependencia más esquinas (E) es una extensión de esta estructura que asume que las esquinas, en nuestro caso las celdas [1,2], [2,1], $[4,5],[5,4]$ tampoco satisfacen la condición de independencia y, por tanto, también deben diferenciarse del resto. Para cada celda en la diagonal y cada esquina, el modelo asume un parámetro específico de homogamia. 


\section{Latin American Research Review}

El modelo de distancia (D) asume que la probabilidad de una unión varía en función de como se aleja de la diagonal, es decir, que cuanto más dispar es la educación del hombre respecto a la de la mujer o viceversa, más improbable es que exista una unión con esas características. Así pues, se espera que el parámetro 1 sea mayor al parámetro 2, el 2 mayor al 3 y así sucesivamente.

Finalmente, la simetría (S) comprueba si el orden de las variables influye en las interacciones de categorías distintas. Si existiera simetría, por ejemplo, las celdas [4,5] y [5,4] mostrarían el mismo nivel de asociación. Para la homogamia educativa, esto significa que la probabilidad de una unión entre personas con distinto nivel de instrucción es independiente del hecho que sea el hombre o la mujer el que tenga mayor o menor nivel educativo. Para comprobar, sin embargo, si existe una tendencia uniforme en uno de los sexos para unirse hacia arriba o hacia abajo calculamos, siguiendo el ejemplo de Mare (1991), el parámetro de asimetría (A).

El cuadro 2 muestra la estructura y resultados de los principales modelos comprobados, de cuyo ajuste informan los estadísticos Likelihood Ratio $\left(\mathrm{G}^{2}\right)$ y Bayesian Information Criterion (BIC) ${ }^{4}$. En ambos casos, cuanto menor es el valor de estos indicadores, mejor es el ajuste y, por lo tanto, mejor es la capacidad explicativa del modelo. La comparación entre países o censos de los BIC no puede realizarse al tratarse de muestras con tamaño distinto. Sin embargo, si que puede comparase el orden en el que los modelos quedan presentados en función de este indicador, es decir, comparar qué modelos funcionan mejor y qué modelos peor en uno y otro país o censos.

Existen diversas alternativas para presentar y comentar los resultados obtenidos mediante la aplicación de modelos loglineales. En este trabajo, por coherencia argumental, hemos decidido clasificar estos modelos en dos grandes bloques. Con el primer bloque, formado por los modelos 2 a 5, damos respuesta a la pregunta: ¿en qué medida las personas se unen de forma homógama, es decir, con personas de su mismo nivel de instrucción? El segundo bloque está formado por los modelos 6 a 9. Estos modelos ponen el acento en la obtención de parámetros para evaluar la vigencia de la hipergamia femenina o hipogámia masculina (parejas en la que la mujer tiene un nivel de instrucción inferior al del hombre).

4. $G^{2}$ es un estadístico de uso común que funciona razonablemente bien para localizar efectos significativos en tablas de contingencia en las que el número de casos no supera los 1.500 (Knoke y Burke, 1980). G² es proporcional al número de casos, razón por la cual, cuando aumenta el tamaño muestral, este indicador da prioridad a los modelos más complejos, complicando su interpretación sin normalmente aportar información relevante. BIC resuelve parcialmente este problema. Para su cálculo utiliza el indicador $\mathrm{G}^{2}$ y los grados de libertad. Se trata de un indicador que mide la eficiencia del modelo al tener en cuenta su capacidad explicativa y los recursos utilizados. 


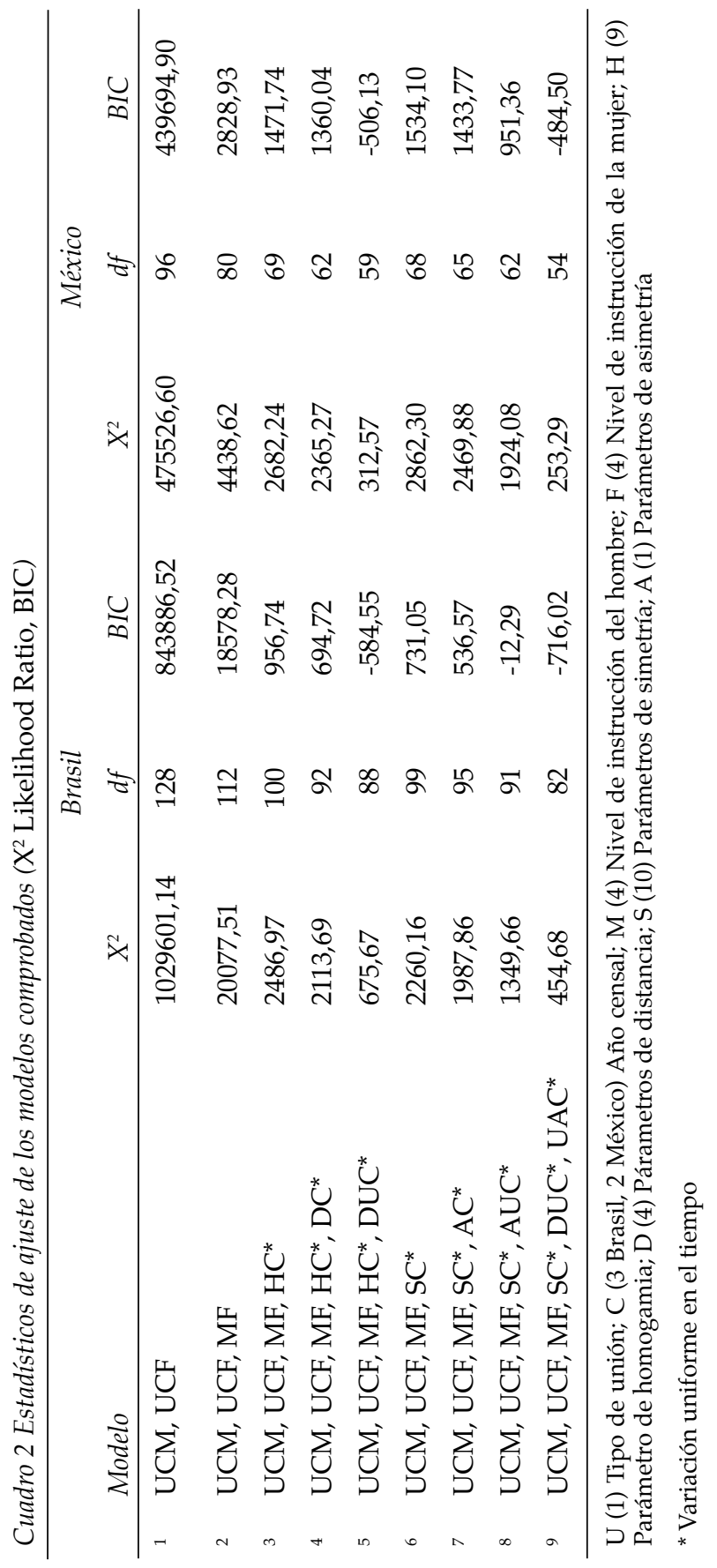




\section{Latin American Research Review}

Ninguna de las hipótesis presentadas tendría razón de ser verificada si, previamente, no comprobamos que la condición de independencia (modelo 1) no se satisface, es decir, que el nivel de instrucción de los individuos es relevante en la selección conyugal y, por tanto, las frecuencias observadas no son el resultado de la combinación azarosa entre los grupos educativos. Bajo el supuesto de independencia, controlados los efectos de la estructura, el riesgo de una unión entre una mujer analfabeta y un hombre con estudios universitarios sería exactamente igual a la de una unión entre universitarios. Pero el deficiente ajuste del modelo 1 en México y Brasil, obliga a rechazar dicho supuesto e invita a explorar nuevas formulaciones que expliquen mejor los datos observados. Se justifica así la razón de ser de los modelos 2 a 9.

\section{Continuidad y cambio de los niveles de homogamia educativa}

El primer bloque de modelos se enfoca básicamente en los niveles específicos de homogamia en función del grupo educativo. El modelo 2 comprueba la siguiente hipótesis: la existencia de una asociación más allá del azar entre el nivel de instrucción de los hombres y el de las mujeres se mantiene constante en el tiempo. El modelo 3 añade a la condición anterior parámetros adicionales para las celdas de la diagonal más esquinas $(\mathrm{H})$ que varían de forma uniforme en el tiempo. Este modelo asume que existe un efecto específico de homogamia en las celdas de la diagonal y en las esquinas. En ambos países, introducir la variación en el tiempo mejora significativamente el ajuste. Las condiciones de homogamia han variado, por tanto, en el tiempo, y la introducción de la dimensión temporal lo corrobora. El modelo 4 añade la matriz de distancias permitiendo que ésta varíe de forma uniforme en el tiempo. El uso combinado de los parámetros de distancia (D) y los de homogamia (H) mejora la capacidad explicativa, puesto que en ambos países, el modelo 4 ajusta mejor que el modelo 3. El modelo 4 mide por separado la distancia educacional entre los cónyuges, independientemente del grupo al que pertenezca cada uno, junto con la asociación específica de cada una de las combinaciones homógamas. En otras palabras, por un lado, se mide el hecho de ser homógamo, y por el otro, el hecho de ser homógamo en función de cada grupo educativo. Sobre la base del modelo 4, el modelo 5 asume que los parámetros de distancia varían no sólo en función del tiempo, sino también en función del tipo de unión. Ésta última consideración hace que mejore sensiblemente el ajuste, hasta alcanzar valores BIC negativos en ambos países.

Del primer bloque de modelos, el modelo 5 ofrece los mejores resultados. De la combinación de los parámetros obtenidos, representamos en la Figura 4 los niveles específicos de homogamia, obtenidos directamente del modelo y expresados en forma de log odds. Los valores superiores a 0 

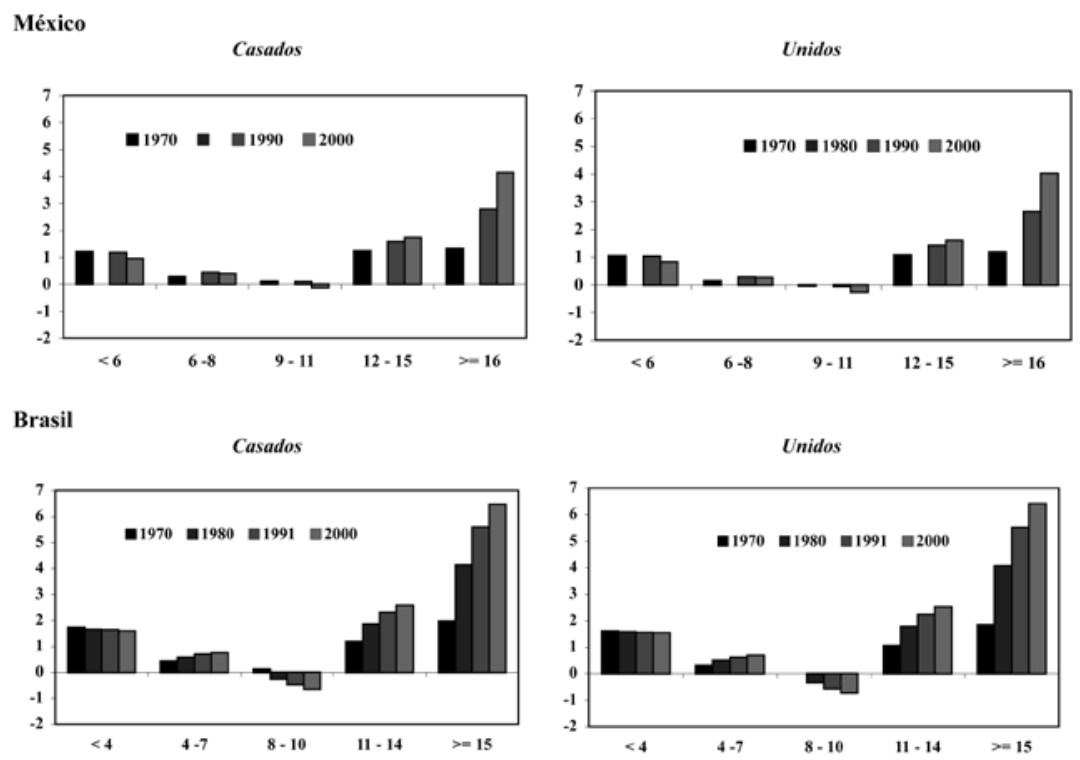

Figura 4 Efectos específicos de homogamia por nivel de instrucción, tipo de unión y país, 1970-2000 ( $\log$ odds del modelo 5)

indican un mayor número de uniones de las que hubiera habido por azar, y los valores inferiores a 0 indican un menor número de uniones respecto a las que hubiera habido por azar. Para saber exactamente cuantas uniones más hay en cada combinación, respecto a las que hubiera habido bajo el supuesto de independencia, es preciso deshacer el logaritmo de los valores representados.

La figura 4 destaca, en primer lugar, que los parámetros de homogamia para todos los grupos educativos, excepto el de la categoría central, son positivos para ambos países, año y tipo de unión. Existe, por tanto, una tendencia manifiesta a formar uniones homógamas. En segundo lugar, la comparación de los parámetros por tipo de unión muestra que no existen diferencias entre estar casado o estar unido y que, por tanto, las conclusiones que se puedan extraer del análisis de los datos son igualmente válidas para ambos tipos de unión. En tercer lugar, la evolución de los niveles de homogamia en uno y otro país presenta pautas similares: aumenta la homogamia entre los grupos más altos, especialmente entre los universitarios, y se mantiene más o menos constante entre los menos instruidos. Es preciso decir, sin embargo, que las categorías extremas suelen presentar niveles de homogamia mayores por la simple razón que tienen limitadas sus opciones matrimoniales en un único sentido, ascendente o descendente. No obstante, este hecho no justifica el aumento de la homogamia entre los universitarios, la cual aumenta de forma importante desde la primera hasta 


\section{Latin American Research Review}

la última generación en México y Brasil en un período donde la población con estudios universitarios también crece. El hecho que los niveles de homogamia entre los universitarios aumenten y también lo haga su tamaño como grupo es una prueba más de la solidez de esta homogamia, puesto que a mayor tamaño, menor puede ser la diferencia entre lo observado y lo esperado en condiciones de independencia.

La homogamia entre los grupos educativos intermedios-primaria y secundaria elemental—es relativamente baja. Este hecho obligaría a reconsiderar en futuros trabajos la pertinencia de incluir las categorías intermedias en nuestra clasificación. En cuarto y último lugar, es importante destacar que existe, pese a las similitudes encontradas, una diferencia notable entre los niveles de homogamia de Brasil y los de México que se observa claramente entre la población universitaria. Los niveles de homogamia son mucho más altos en Brasil que en México y esto significa que las oportunidades para la movilidad social a partir de la educación son mucho menores, que los grupos están más cerrados, que la dificultad de unirse fuera de su grupo es mucho mayor, y que, en definitiva, las desigualdades sociales están más acentuadas en Brasil que en México.

\section{Hipergamia femenina}

Habiendo constatado la tendencia mayoritaria a formar pareja en modo homogámico, en la segunda etapa de este análisis nos interrogamos sobre las parejas que se constituyen entre hombres y mujeres de distinto nivel de instrucción. Nuestro interés principal respecto a estas parejas se concentra en analizar si las pautas de heterogamia masculina y femenina son equivalentes. En los casos de México y Brasil para 1970 y 1980, la proporción de mujeres que han formado pareja hipergámicamente, es decir, con un cónyuge más instruido que ellas, es superior a la proporción de las que lo han hecho hipogámicamente. Debemos preguntarnos, sin embargo, ¿en qué medida esta pauta de hipergamia educativa femenina es producto de las diferencias educativas entre hombres y mujeres?

El modelo 6 asume que las pautas de interacción entre cónyuges son simétricas y que varían uniformemente en el tiempo. La simetría asume que la probabilidad de una unión entre dos personas con distinto nivel educativo no está afectada por el hecho de que sea el hombre o la mujer el que tenga mayor o menor nivel. El modelo 7 añade un parámetro adicional, variable en el tiempo, a esta condición. Se trata de un parámetro de asimetría que permite una tendencia uniforme de las mujeres a unirse hacia arriba o hacia abajo (hipergamia o hipogamia respectivamente) para todas las combinaciones de niveles educativos (Mare, 1991, p. 22). El modelo 8 asume que el parámetro de asimetría también varía en función del tipo de unión. El indicador BIC es altamente sensible a esta 
México

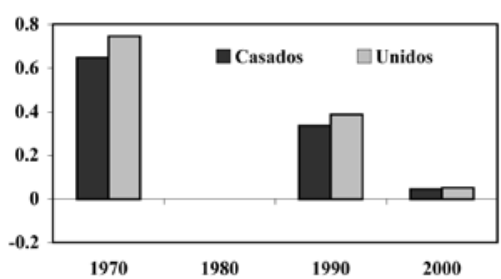

Brasil

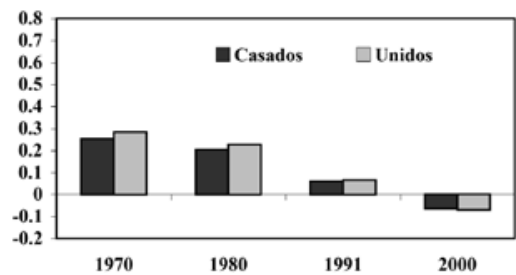

Figura 5 Efectos específicos de hipergamia femenina por tipo de unión y país, 1970-2000 ( $\log$ odds del modelo 9)

ligera modificación, prueba suficiente para demostrar que los parámetros añadidos están captando patrones importantes. El modelo 9 añade la estructura de distancias, tal y como aparece en el modelo 5 del bloque anterior. La introducción de la distancia es fundamental para que mejore ostensiblemente la calidad del modelo. Con el modelo 9, el BIC alcanza tanto en México como en Brasil valores negativos. Utilizamos el parámetro de asimetría que nos proporciona este modelo como medida sintética e ilustrativa de cómo ha variado el modelo tradicional de hipergamia femenina (Figura 5). La interpretación de este parámetro es análoga a la de los parámetros de la Figura 4: los valores positivos indican una desviación positiva respecto al escenario de independencia, y los valores negativos indican lo contrario. Como se puede observar, las Figuras 4 y 5 no comparten la misma escala, razón por la cual los resultados no son directamente comparables, lo que puede dar una falsa impresión en la interpretación de los resultados. Sin embargo, la utilización de una escala distinta se justifica por el siguiente motivo: el parámetro de hipergamia afecta a todas las combinaciones hipérgamas de la tabla, es decir, a 10 de las 25 combinaciones posibles entre la educación del hombre y de la mujer (ver Cuadro 1). En cambio, los parámetros específicos de homogamia representan a una sola combinación de la tabla, a una sola celda. Los efectos de hipergamia son menores a los de homogamia pero afectan a un número mayor de celdas. Consideramos, pues, que los parámetros de las Figuras 4 y 5 tienen lógicas diferenciadas, hecho que justifica la utilización de una escala distinta.

Libre del efecto de la distribución marginal de hombres y mujeres por nivel educativo, el parámetro de asimetría indica que la hipergamia femenina ha perdido vigencia en ambos países en el periodo analizado. En el caso de México, la hipergamia se reduce significativamente, pero sigue siendo positiva en el año 2000. En el caso de Brasil, la hipergamia se reduce hasta alcanzar valores negativos en el año 2000, invirtiendo la pauta observada hasta el momento. De forma sistemática para todos los 
años y países, la hipergamia femenina es más común entre las uniones consensuales que entre los matrimonios, aunque esta diferencia tiende a desaparecer. Como en el caso de la homogamia por nivel de instrucción, la diferencia entre Brasil y México es de nivel: la hipergamia femenina es mayor en México que en Brasil. La desigualdad de género ha sido y sigue siendo más importante en México que en Brasil.

\section{CONCLUSIONES}

Las transformaciones en la estructura por nivel de estudios de la población mexicana y brasileña acontecidas en las últimas tres décadas son de tan vasta proporción que su alusión es obligada en primer lugar. La población sin estudios ha disminuido a una tercera parte de los niveles de 1970, y la población universitaria ha aumentado significativamente en ambos países. Pese a esta expansión, en el año 2000 una quinta parte de la población observada no había finalizado sus estudios primarios, lo que demuestra que las desigualdades sociales, y en México también de género, siguen reproduciéndose a través de un acceso desigual a la educación. Ante este escenario, las pautas $\mathrm{y}$ tendencias en homogamia educativa observadas, tanto en los matrimonios como en las uniones consensuales, no contribuyen a reducir tales desigualdades. Pese a la disminución de las parejas homógamas como consecuencia de la diversificación de la estructura educativa, el análisis de los niveles específicos de homogamia muestra que existe para todos los niveles educativos una propensión manifiesta a unirse dentro del mismo grupo, especialmente entre las capas más instruidas. El aumento de la homogamia educativa entre los universitarios corrobora la hipótesis con la que iniciábamos este artículo: voluntad y oportunidad se alían con los más instruidos para unirse entre ellos. Pero cuando el acceso a la educación universitaria es exclusivo de las clases pudientes, el aumento de la homogamia entre los universitarios, a diferencia de la interpretación que se da en países más desarrollados, puede ser más bien el resultado de una homogamia por clase social más potente que la educativa.

Las diferencias de género entorno a cómo hombres y mujeres incorporan la educación en la selección conyugal se reducen. La pauta tradicional de hipergamia femenina pierde vigencia durante este periodo hasta llegar a invertirse en Brasil para el año 2000. En México, no obstante, el modelo de hipergamia femenina es aún vigente aunque su presencia es significativamente más baja que en 1970. Este cambio de patrón es a su vez coherente con la otra hipótesis planteada en la introducción: se observará una tendencia hacia una mayor simetría entre hombres y mujeres en la influencia que la educación ejerce en la configuración de las preferencias conyugales. 
Más allá de los resultados de homogamia educativa, esta investigación ha puesto de manifiesto la utilidad de los microdatos censales para llevar a cabo comparaciones internacionales. Por su periodicidad, sus objetivos y su diseño, los censos de población comparten un amplio número de características que posibilitan la comparación de sus datos, más aún cuando se disponen de microdatos. Los microdatos permiten obtener mayor provecho de los censos y adaptar los datos según convenga a las necesidades de la investigación para poder llevar a cabo tratamientos estadísticos que posibiliten la obtención de resultados comparables. La base de datos IPUMS-International facilita el acceso a los microdatos con un valor añadido: su armonización. En los próximos años, los censos de la mayoría de los países de América Latina estarán disponibles en Internet gracias al proyecto IPUMS-América Latina. Los resultados ahora encontrados para México y Brasil podrán ser validados para el conjunto de estos países, entre ellos Chile, Costa Rica, Ecuador y Venezuela, en una primera etapa, y luego Argentina, Bolivia, República Dominicana, El Salvador, Guatemala, Honduras, Nicaragua, Panamá, Paraguay, Perú, Puerto Rico y Uruguay. De todos los institutos de estadística de América Latina, sólo la Oficina Nacional de Estadística de Cuba no participa en el proyecto. La extensión de este trabajo al resto de países de América Latina es, por tanto, una vía natural de continuidad de esta investigación. Por un lado, conoceríamos mejor la realidad de cada país y, por otro, compararíamos las experiencias entre ellos. En el caso de México y Brasil, la comparación ha permitido observar que pese a la coincidencia en las pautas y tendencias en homogamia educativa, existen aspectos diferenciales que matizan y enriquecen el análisis, y que muestran otros factores de importancia en el estudio de la homogamia. La comparación ha permitido ver que la desigualdad de género tiene especial importancia en el caso de México y que las barreras educacionales en Brasil son más elevadas que en México.

Ahora bien, en este trabajo no hemos introducido elementos para demostrar de forma integrada una relación de causalidad entre nuestras hipótesis iniciales y los resultados encontrados. Con datos específicos sobre los retornos a la educación, la incorporación de la mujer a la actividad remunerada, la desigualdad de género en materia de ingresos por trabajo, la evolución de los indicadores macroeconómicos, o la edad al primer matrimonio podríamos determinar con mayor precisión la relación que estos indicadores tienen con la evolución de la homogamia educativa. Asimismo, la distribución territorial de la población por nivel educativo y las oportunidades que esta genera para las uniones mixtas, la importancia del lugar de trabajo como mercado matrimonial, y la transición hacia sociedades claramente urbanas son aspectos a tener también en cuenta en futuras investigaciones. En efecto, la incorporación de variables como el lugar y ámbito de residencia, la edad al 
matrimonio, o la condición social de los padres y madres facilitarían la comprobación de hipótesis más ambiciosas entorno a las causas que explican las pautas de homogamia educativa descritas en este trabajo. En el caso particular de Brasil, la raza debe ser tomada en consideración para responder a ¿cómo la educación, variable adquirida por excelencia, interactúa con la raza? ¿Cómo se relaciona la homogamia educativa con la endogamia racial? ¿Son las diferencias raciales atribuibles exclusivamente a las diferencias en el nivel educativo medio que tiene la población de una u otra raza?

Finalmente, también será necesario evaluar las consecuencias que el aumento de la homogamia tendrá en la reproducción de las desigualdades sociales. Si la posición social determina el acceso a la educación y los distintos grupos adoptan una práctica homógama, esto no hará más que reforzar las desigualdades y limitar las oportunidades para la movilidad social. Pero no debemos olvidar, no obstante, que si bien los niveles de homogamia en la cúspide de la jerarquía educativa han aumentado en las últimas décadas, también lo ha hecho la población con estudios universitarios. Las consecuencias, por tanto, que el aumento de los niveles de homogamia pueda tener en la exacerbación de las desigualdades sociales, han sido contrarestadas por una importante expansión educativa, fruto de la actitud y actuación de los poderes públicos en materia educativa, acompañadas de crecimiento económico.

\section{REFERENCIAS}

Becker, Gary

1974 "A Theory of Marriage," in Economics of the Family, ed. T.W.Schultz (ChicagoLondon: University of Chicago Press), 299.

Birkelund, Gunn E., and Johan Heldal

2003 "Who Marries Whom? Educational Homogamy in Norway." Demographic Research 8 (1).

Blackwell, Debra L.

1998 "Marital Homogamy in the United States: The Influence of Individual and Paternal Education." Social Science Research 27: 159.

Esteve, Albert

2005 “Tendencias en homogamia educacional en México: 1970-2000." Estudios Demográficos y Urbanos 59: 341.

Esteve, Albert, and Clara Cortina

2006 "Changes in Educational Assortative Mating in Contemporary Spain." Demographic Research 14: 405.

García, Brígida, and Orlandina de Oliveira

1994 Trabajo femenino y vida familiar en México. Ciudad de México: El Colegio de México.

Halpin, Brendan, and Tak Wing Chan

2003 "Educational Homogamy in Ireland and Britain: Trends and Patterns." British Journal of Sociology 54: 473.

Hoffman, Rodolfo, and Eugenia Troncoso

2004 "Participação da mulher no mercado de trabalho e desigualdade da renda domiciliar per capita no Brasil: 1981-2002." Nova Economia Belo Horizonte 14 (2): 45. 
Hout, Michael

1982 "The Association between Husbands' and Wives' Occupation in Two-earner Families." American Journal of Sociology 87: 397.

Instituto Brasileiro de Geografia e Estadística (IBGE)

$1970 \quad$ Mostra do VIII Recenseamento Geral do Brasil. Censo Demográfico 1970. Rio de Janeiro.Instituto Nacional de Estadística, Geografía e Informática (INEGI), 2000 Muestra del XII Censo General de Población y Vivienda de la República de México, 2000. Aguascalientes: Ags.

1980 Mostra do IX Recenseamento Geral do Brasil. Censo Demográfico 1980. Rio de Janeiro.

1990 Mostra do X Recenseamento Geral do Brasil. Censo Demográfico 1991. Rio de Janeiro.

2000 Mostra do XI Recenseamento Geral do Brasil. Censo Demográfico 2000. Rio de Janeiro.

Instituto Nacional de Estadística, Geografía e Informática (INEGI)

1970 Muestra del IX Censo General de Población y Vivienda de la República de México, 1970. Aguascalientes, Ags.

1990 Muestra del XI Censo General de Población y Vivienda de la República de México,

Johnson, Robert A. 1990. Aguascalientes, Ags.

1980 Religious Assortive Mating in the United States (New York: Academic Press).

Kalmijn, Matthijs

1998 "Intermarriage and Homogamy: Causes, Patterns, Trends." Annual Review of Sociology 24: 395.

Knoke, David, and Peter J. Burke

1980 Log Linear Models (Beverly Hills: Sage Publications).

Mare, Robert D.

1991 "Five Decades of Assortative Mating." American Sociological Review 56: 15.

Marteleto, Letícia J.

2004 "Desigualdade intergeracional de oportunidades educacionais: Una analise de matrícula e escolaridade das crianças brasileiras" (244, Belo Horizonte: UFMG/CEDEPLAR).

McCaa, Robert

1993 "Gender in the Melting Pot: Marital Assimilation in New York City, 1900-1980." The Journal of Interdisciplinary History 24: 2.

McPherson et al., Miller L.

2001 "Birds of a Feather: Homophily in Social Networks." Annual Review of Sociology 27: 415 .

Mier et al., Marta

2003 "Inequalities in Mexican Children's Schooling." Journal of Comparative Family Studies 34: 435.

Minnesota Population Center

2006 Integrated Public Use Microdata Series-International: Version 2.0. Minneapolis: University of Minnesota.

Oppenheimer, Valerie K.

$1988 \quad$ "A Theory of Marriage Timing." The American Journal of Sociology 94: 563.

Pagnini, Deanna L., and S. Philip Morgan

$1990 \quad$ "Intermarriage and Social Distance among U.S. Immigrants at the Turn of the Century." American Sociological Review 96: 405.

Parker, Susan W., and Carla Pederzini

1999 "Gender Differences in Education in México," in The Economics of Gender in Mexico, E.G. Katz and M.C. Correia, eds. Washington: The World Bank, 9.

Parrado, Emilio A., and René M. Zenteno

2002 "Gender Differences in Union Formation in México: Evidence from Marital

Qian, Zenchao Search Models." Journal of Marriage and Family 64: 756.

1998 "Changes in Assortative Mating: The Impact of Age and Education." Demography 35: 279. 


\section{Latin American Research Review}

Quilodrán, Julieta

1993 "Cambios y permanencias de la nupcialidad en México." Revista Mexicana de Sociología 55: 17.

Schwartz, Christine, and Robert D. Mare

2005 "Trends in Educational Assortative Marriage from 1940 to 2003." Demography 42: 621 .

Smits, Jeroen

2003 "Social Closure among the Higher Educated: Trends in Educational Homogamy in 55 Countries." Social Science Research 32: 251.

Wajnman, Simone, and Eduardo L.G. Rios-Neto

2001 "Women's Participation in the Labor Market in Brazil: Elements for Projecting Levels and Trends." Brazilian Journal of Population Studies 2: 41. 
Apéndice 1 México: Distribución de las parejas por tipo de unión, año, nivel de instrucción de los hombres y nivel de instrucción de las mujeres

\begin{tabular}{|c|c|c|c|c|c|}
\hline Hombre/Mujer & Menos de 6 & 6 a 8 & 9 a 11 & 12 a 15 & $160+$ \\
\hline \multicolumn{6}{|l|}{ Casados } \\
\hline \multicolumn{6}{|l|}{1970} \\
\hline Menos de 6 & 7145 & 1258 & 83 & 60 & 36 \\
\hline 6 a 8 & 1664 & 2180 & 166 & 118 & 16 \\
\hline 9 a 11 & 162 & 529 & 158 & 101 & 16 \\
\hline 12 a 15 & 84 & 317 & 105 & 159 & 15 \\
\hline $16 o+$ & 55 & 325 & 164 & 217 & 83 \\
\hline \multicolumn{6}{|l|}{1990} \\
\hline Menos de 6 & 13000 & 3317 & 577 & 61 & 34 \\
\hline 6 a 8 & 4162 & 6410 & 1759 & 201 & 72 \\
\hline 9 a 11 & 1093 & 3015 & 3153 & 418 & 235 \\
\hline 12 a 15 & 186 & 849 & 1502 & 698 & 232 \\
\hline $160+$ & 94 & 654 & 2073 & 893 & 1634 \\
\hline \multicolumn{6}{|l|}{2000} \\
\hline Menos de 6 & 68073 & 32411 & 10834 & 2213 & 414 \\
\hline 6 a 8 & 34530 & 62057 & 30824 & 8859 & 1700 \\
\hline 9 a 11 & 14137 & 40970 & 60918 & 24514 & 5454 \\
\hline 12 a 15 & 3251 & 13328 & 30665 & 37698 & 11243 \\
\hline $160+$ & 917 & 4293 & 15150 & 29834 & 36458 \\
\hline \multicolumn{6}{|l|}{ Unidos } \\
\hline \multicolumn{6}{|l|}{1970} \\
\hline Menos de 6 & 1156 & 100 & 10 & 1 & 7 \\
\hline 6 a 8 & 205 & 104 & 6 & 3 & 2 \\
\hline 9 a 11 & 13 & 25 & 4 & 1 & 1 \\
\hline 12 a 15 & 11 & 10 & 1 & 3 & 1 \\
\hline $16 o+$ & 11 & 6 & 1 & 2 & 1 \\
\hline \multicolumn{6}{|l|}{1990} \\
\hline Menos de 6 & 3085 & 601 & 112 & 14 & 9 \\
\hline 6 a 8 & 846 & 717 & 190 & 22 & 5 \\
\hline 9 a 11 & 238 & 310 & 221 & 28 & 13 \\
\hline 12 a 15 & 39 & 80 & 88 & 35 & 12 \\
\hline $160+$ & 18 & 38 & 74 & 38 & 38 \\
\hline \multicolumn{6}{|l|}{2000} \\
\hline Menos de 6 & 24395 & 10404 & 3815 & 690 & 85 \\
\hline 6 a 8 & 12370 & 16335 & 7795 & 1979 & 382 \\
\hline 9 a 11 & 5603 & 10703 & 11315 & 3438 & 690 \\
\hline 12 a 15 & 1475 & 3286 & 5272 & 3983 & 1041 \\
\hline
\end{tabular}


84 Latin American Research Review

Apéndice 2 Brasil: Distribución de las parejas por tipo de unión, año, nivel de instrucción de los hombres y nivel de instrucción de las mujeres

\begin{tabular}{|c|c|c|c|c|c|}
\hline Hombre/Mujer & Menos de 4 & $4 a 7$ & 8 a 10 & 11 a 14 & $150+$ \\
\hline \multicolumn{6}{|l|}{ Casados } \\
\hline \multicolumn{6}{|l|}{1970} \\
\hline Menos de 4 & 168931 & 19849 & 722 & 531 & 9 \\
\hline 4 a 7 & 24527 & 39251 & 2930 & 2813 & 137 \\
\hline 8 a 10 & 1455 & 5345 & 2660 & 1774 & 167 \\
\hline 11 a 14 & 501 & 3530 & 2411 & 3416 & 347 \\
\hline 15 o + & 94 & 947 & 1549 & 3004 & 1096 \\
\hline \multicolumn{6}{|l|}{1980} \\
\hline Menos de 4 & 136611 & 33980 & 2419 & 1559 & 114 \\
\hline 4 a 7 & 34677 & 60286 & 7729 & 6561 & 828 \\
\hline 8 a 10 & 2983 & 10436 & 6109 & 4614 & 823 \\
\hline 11 a 14 & 1057 & 6543 & 5282 & 10170 & 2640 \\
\hline $15 \mathrm{o}+$ & 162 & 1514 & 2396 & 8088 & 6362 \\
\hline \multicolumn{6}{|l|}{1990} \\
\hline Menos de 4 & 66063 & 30374 & 3959 & 2701 & 199 \\
\hline 4 a 7 & 23277 & 54690 & 12374 & 9359 & 1066 \\
\hline 8 a 10 & 3047 & 12148 & 10152 & 8074 & 1301 \\
\hline 11 a 14 & 1245 & 6663 & 8578 & 19394 & 5154 \\
\hline 15 o + & 148 & 836 & 1858 & 8604 & 9609 \\
\hline \multicolumn{6}{|l|}{2000} \\
\hline Menos de 4 & 40055 & 31354 & 5962 & 4301 & 346 \\
\hline 4 a 7 & 20590 & 62200 & 19460 & 15967 & 1462 \\
\hline 8 a 10 & 4006 & 18173 & 17038 & 15585 & 2001 \\
\hline 11 a 14 & 2043 & 10191 & 13990 & 36664 & 8505 \\
\hline 15 o + & 165 & 751 & 1676 & 10327 & 11865 \\
\hline
\end{tabular}

Unidos

1970

$\begin{array}{lrrrrr}\text { Menos de } 4 & 16004 & 1123 & 31 & 14 & 3 \\ 4 \text { a } 7 & 2528 & 1635 & 84 & 54 & 8 \\ 8 \text { a } 10 & 226 & 258 & 81 & 33 & 6 \\ 11 \text { a } 14 & 74 & 152 & 62 & 60 & 8 \\ 15 \text { o }+ & 25 & 54 & 66 & 47 & 16 \\ 1980 & & & & & \\ \text { Menos de } 4 & 23910 & 4553 & 372 & 135 & 27 \\ 4 \text { a } 7 & 6404 & 6408 & 872 & 464 & 54 \\ 8 \text { a } 10 & 841 & 1449 & 626 & 319 & 63 \\ 11 \text { a } 14 & 226 & 695 & 443 & 595 & 134 \\ 15 \text { o }+ & 46 & 162 & 204 & 373 & 356\end{array}$


HOMOGAMIA EDUCACIONAL EN MÉXICO Y BRASIL 85

Appendice 2 (concluido)

\begin{tabular}{lrrrrr} 
Hombre/Mujer & Menos de 4 & 4 a 7 & 8 a 10 & 11 a 14 & $15 o+$ \\
\hline Casados & & & & & \\
$\mathbf{1 9 9 0}$ & & & & & \\
$\quad$ Menos de 4 & 21616 & 7611 & 1159 & 537 & 50 \\
4 a 7 & 7585 & 10736 & 2521 & 1415 & 179 \\
8 a 10 & 1298 & 3058 & 1890 & 1129 & 174 \\
11 a 14 & 538 & 1623 & 1422 & 2211 & 577 \\
15 o + & 62 & 225 & 302 & 902 & 778 \\
$\mathbf{2 0 0 0}$ & & & & & \\
Menos de 4 & 23780 & 13012 & 2405 & 1193 & 113 \\
4 a 7 & 10259 & 18949 & 5360 & 3142 & 215 \\
8 a 10 & 2033 & 5624 & 3907 & 2577 & 226 \\
11 a 14 & 939 & 2930 & 2869 & 4456 & 729 \\
15 o + & 73 & 209 & 278 & 904 & 727 \\
\hline
\end{tabular}

